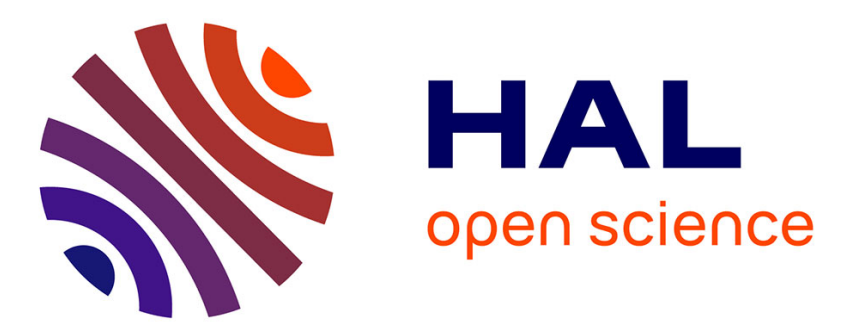

\title{
Molecular Simulation of Sorption-Induced Deformation in Atomistic Nanoporous Materials
}

Mingyang Chen, Benoit Coasne, Robert Guyer, Dominique Derome, Jan

Carmeliet

\section{- To cite this version:}

Mingyang Chen, Benoit Coasne, Robert Guyer, Dominique Derome, Jan Carmeliet. Molecular Simulation of Sorption-Induced Deformation in Atomistic Nanoporous Materials. Langmuir, 2019, 35 (24), pp.7751-7758. 10.1021/acs.langmuir.9b00859 . hal-02328658

\section{HAL Id: hal-02328658 \\ https://hal.science/hal-02328658}

Submitted on 5 Nov 2020

HAL is a multi-disciplinary open access archive for the deposit and dissemination of scientific research documents, whether they are published or not. The documents may come from teaching and research institutions in France or abroad, or from public or private research centers.
L'archive ouverte pluridisciplinaire HAL, est destinée au dépôt et à la diffusion de documents scientifiques de niveau recherche, publiés ou non, émanant des établissements d'enseignement et de recherche français ou étrangers, des laboratoires publics ou privés. 


\title{
Molecular simulation of sorption-induced deformation in atomistic nanoporous materials
}

\author{
Mingyang Chen ${ }^{1}$, Benoit Coasne ${ }^{2, *}$, Robert Guyer ${ }^{3}$, Dominique Derome ${ }^{4}$ and Jan \\ Carmeliet $^{1, *}$
}

${ }^{1}$ Chair of Building Physics, Department of Mechanical and Process Engineering, ETH Zurich, 8093 Zurich, Switzerland

${ }^{2}$ Univ. Grenoble Alpes, CNRS, LIPhy, 38000 Grenoble, France

${ }^{3}$ Department of Physics, University of Nevada, Reno, 1664 N. Virginia Street, 89557 Nevada, United States

4 Laboratory for Multiscale Studies in Building Physics-Swiss Federal Laboratories for Materials Science and Technology, Ueberlandstrasse 129, 8600 Duebendorf, Switzerland It is not formated for langmuir - see affiliations

* Correspondence and requests should be addressed to B. C. (benoit.coasne@univ-grenoblealpes), J. C. (cajan@ethz.ch) 


\begin{abstract}
An atomistic slit pore model is built to study the sorption-induced deformation of nanoporous materials with the help of molecular simulation. Both sorption and strain isotherms are determined to probe anisotropic deformation behaviors induced upon gas adsorption. A detailed analysis shows that the driving microscopic mechanisms at different sorption stages are different. At high relative pressure, as expected from the classical macroscopic picture, the pore deformation is governed by Laplace pressure as the porosity gets filled with liquid due to capillary condensation. In such situation, the strain can be predicted from the corresponding stiffness modulus. At low pressure, when liquid films are adsorbed at the pore surfaces and separated by the vapor phase, the strain is driven by the attractive solid-fluid forces and 2D pressure within the film, and the deformation is confined in the direction parallel to the film-solid interface. Analysis of isosteric heat of adsorption shows that the contribution arising from the deformation is small compared to the sorption contribution, which indicates that the influence of deformation on the sorption process is limited. I have a few concerns with the TOC figure: A. When converted to the real sizes for TOC, I m not sure "Pressure" and "deformation" are readable. B. The curved menisci do not look hemispherical to me. I understand that there is the effect of the adsorbed film but still the central part of the menisci should appear hemispherical
\end{abstract}

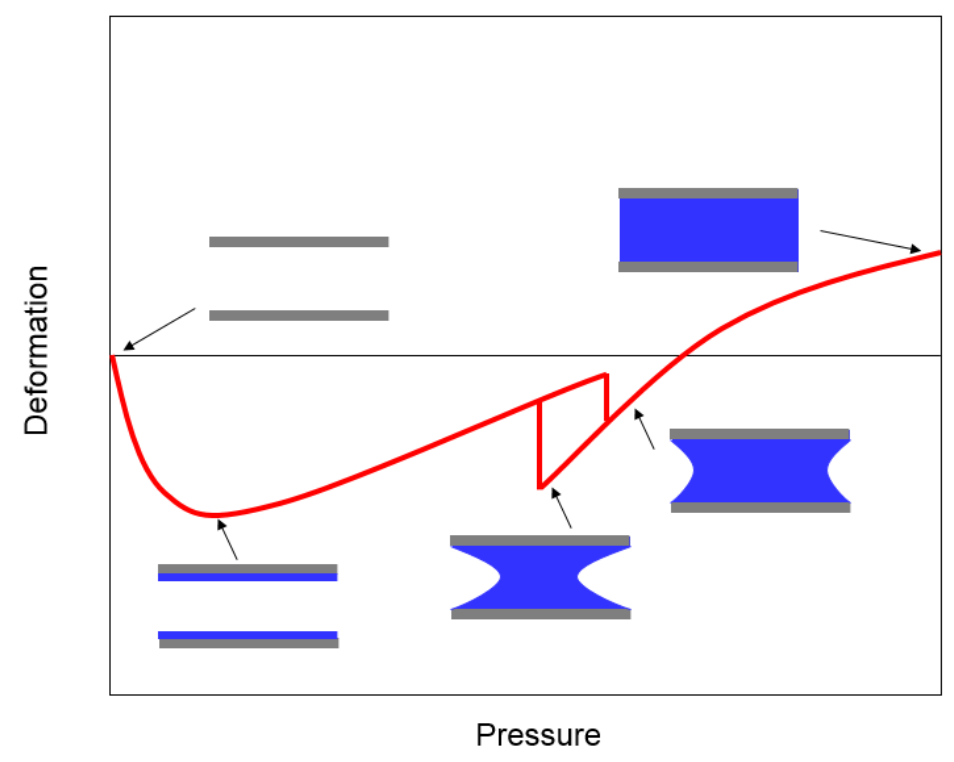




\section{Introduction}

When using superscirpt referernces, there is no space betweeb the reference and the word before Sorption-induced deformation is a common phenomenon in various types of porous materials ${ }^{1}$. This phenomenon arises at the molecular level because of the strong intermolecular forces at the sorption surface induced by the adsorbent-adsorbate interactions ${ }^{23}$. Specifically, for nanoporous materials, experimental and theoretical studies find sorption-induced deformation generally associated with first order phase transitions which correspond to capillary condensation and evaporation ${ }^{4}$. As a result, the capillary hysteresis usually observed in sorption isotherms is accompanied by hysteresis in strain isotherms ${ }^{5}$. Moreover, it is found that the deformation has distinct characteristics before and after phase transition ${ }^{6,7}$, which indicates that the sorption-induced deformation is governed by different mechanisms at different adsorption stages.

When you have to rferences; they should be separated by commas. For instance 9,10 not 910. From a mechanical perspective, sorption-induced deformation of nanoporous materials is generally attributed to either disjoining pressure ${ }^{8}$, surface stress ${ }^{910}$ or Laplace pressure ${ }^{6}$ effects. In the formulat it should be W(e) not W alone. Also, w should be w(e) etc. The disjoining pressure is defined as $\omega=\mathrm{d} W / \mathrm{d} e$, where $W(e)$ is the surface potential that stabilizes the adsorbed film of a thickness $e$. Note that this definition indicates that disjoining pressure acts in the direction perpendicular to the surface. However, it must be recalled that the surface potential term scales with the film surface area (which pertains to the direction parallel to the pore surface). The concept of disjoining pressure is often used to explain sorption-induced deformation. Gor and Neimark ${ }^{11}$ use the disjoining pressure concept as formalized in the Derjaguin-Broekhoff-de Boer (DBdB) theory to explain the deformation of cylindrical pores during sorption before the occurrence of capillary condensation. A similar approach is adopted by Kolesnikov et al ${ }^{12}$ when considering sorption in a pore network. The surface free energy of the liquid-vapor interface is written as dependent on the liquid film thickness through the use of the disjoining pressure term. On similar systems, the concept of disjoining pressure is also used in computational modeling work using methods such as Grand Canonical Monte Carlo (GCMC) and density 
functional theory DFT. For example, conducting GCMC simulations, Cornette et al ${ }^{13}$ find that adsorption-induced deformation in a slit pore is driven by the solvation pressure which is equivalent to the disjoining pressure. Similar observation is found in Grégoire et al. who used DFT calculations to describe the mechanics and physics of nanoconfined fluids ${ }^{14}$.

A second explanation of sorption-induced deformation stems from the work of Bangham ${ }^{15}$ which was extended by Shuttleworth ${ }^{16}$. In this approach, the surface stress $\sigma_{S}^{A}$ can be expressed according to Shuttleworth equation as $\sigma_{S}^{A}=\gamma_{S}^{A}+\mathrm{d} \gamma_{S}^{A} / \mathrm{d} \varepsilon_{A}$, where $\gamma_{S}^{A}$ is the surface energy and $\varepsilon_{A}$ is the surface strain. Surface stress arises from the spreading effect of free energy of the solid-liquid interface and the dependence on strain. Unlike disjoining pressure, surface stress acts within the interface and drives the deformation along the inplane direction of the surface. Zhang ${ }^{17}$ showed that the swelling of coal subjected to pure $\mathrm{CO}_{2}$ and mixtures of $\mathrm{CO}_{2} / \mathrm{CH}_{4}$ injections can be modeled assuming that all deformation is due solely to surface stress. Furthermore, as indicated by Gor et al. ${ }^{10}$, the surface stress can be either positive or negative so that both swelling and shrinkage of the solid can be observed. A third explanation of sorption-induced deformation relies on the Laplace pressure, which characterizes the pressure difference across a curved boundary between a vapor and a liquid. The Laplace pressure is generally invoked to explain the deformation occurring after capillary condensation and in the presence of a liquid meniscus at the open end of the pore.

The three different mechanisms mentioned above can have combined effects on the deformation. As a result, a comprehensive description of adsorption-induced deformation should address all these mechanisms. A reasonable and straightforward approach to conduct such analysis is to use a simple pore geometry such as the slit pore model. The major advantage of this pore model over other geometries is that one can easily separate contributions from the directions perpendicular and parallel to the adsorption surface (which involve disjoining pressure and surface stress, respectively). Studies of sorption behavior using the slit pore model date back to the 1950s when Steele and Halsey ${ }^{18}$ 
modified the theory of the interaction of gas molecules with a structureless plane to treat capillary space. In the same spirit, Stoeckli ${ }^{19}$ calculated the adsorption potential for a slit pore. Derjaguin and Churaev ${ }^{20}$ addressed capillary condensation in slit pores with the concept of disjoining pressure. However, disjoining pressure is here used solely to model phase transition, while the related mechanical effect is not considered. In addition to such analytical studies, sorption behavior is also extensively studied using numerical simulations. These studies ${ }^{21-25}$ show that the hysteresis observed in the sorption isotherm strongly depends on the pore width and surface energy heterogeneity. Few studies focus on the sorption-induced deformation in slit pores by looking at the in-pore pressure. It was shown by Long et al ${ }^{26-28}$ with GCMC method that the in-pore pressure is strongly enhanced by confinement of nanopores. As the result, the in-pore pressure, in both normal and tangential directions, can be several orders of magnitude larger than the bulk phase pressure in equilibrium. This high in-pore pressure indicates that deformation induced by sorption in nanoporous material can be significant, and the high sensitivity of the in-pore pressure over the bulk pressure shows that the variation of deformation can be large during the sorption process. Moreover, the results by Long et al also show that the pressure tensor differs in normal direction and longitudinal direction, indicating an anisotropic deformation pattern. Similar high in-pore pressure and related deformation are also given by other works employing either GCMC method ${ }^{2930}$ or density functional theory ${ }^{14} 31$. However, a great limitation of works stated above is that the pore systems built are not deformable, thus one can only deduce the deformation by assuming a mechanical constitutive relationship (Hooke's law for instance). A deformable slit pore model was considered by Schoen et al ${ }^{32-35}$ where the porosity is located between two Einstein solid substrates (i.e. each atom is attached to its original position through a harmonic potential). While giving good results for the normal strain, Einstein solids cannot model the deformation along the longitudinal direction properly. Indeed, as each solid atom is connected to a fixed point instead of neighboring atoms, the longitudinal stress depends on the absolute displacement instead of the relative displacement between neighboring atoms so non-physical strain fields are obtained. 
While many experimental and theoretical studies have been conducted on the sorptioninduced deformation of nanoporous materials, most research has been limited to a single deformation mechanism out of the three at play in real materials. In this paper, we aim at characterizing the contributions of these different mechanisms in sorption-induced deformation with the help of a realistic slit pore model described at the molecular scale. The hysteresis observed in sorption and deformation at the single pore level is also discussed.

\section{Models and Methods}

\section{If for langmuir (or equivalent) you need to have subsections numbering as well Preparation of the solid model}

In this paper, we build a slit pore model composed of two atomic solid substrates (see Figure 1). Each substrate, which is made up of two layers of solid atoms, is first generated in a simple cubic crystal with a lattice parameter $\mathrm{a}=0.3822 \mathrm{~nm}$. To avoid crystallization of the confined liquid that would be induced by the use of a crystalline solid surface, amorphization of the subtrates is achieved by applying a random spatial displacement in the $\mathrm{x}-\mathrm{y}$ plane to each solid atom. Note that the solid surface remains plane as no extra roughness is introduced. Then, a harmonic bond is added between each pair of nearest neighboring solid atoms to mimic a covalent chemical bond as in real solids. To further reduce the problem complexity, we classify all the bond lengths modified into 20 length bins and replace each with the average length of its corresponding bin. This allows describing the system by specifying just 20 different harmonic bonds instead of specifying parameters for every bond length. However, the replacement introduces pre-stresses into the substrates which need to be relaxed before sorption simulation. Specifically, the solid model is relaxed at constant temperature $87 \mathrm{~K}$ using a Molecular Dynamics (MD) run in the NVT ensemble. Using the strategy above, one can produce any desired stiffness of the 
substrate by tuning the bond strength. In this work, the stiffness of the solid substrates is set to $10 \mathrm{GPa}$.

As for the longitudinal direction, the substrates are continuous solid materials with a stiffness which solely depends on the bond strengths. I don't undertstand the previous sample: why do you say that it s continuous? If it s made of atoms, it s not really continuuous. As for the normal direction, the stiffness arises from the surrounding nanoporous void which provides a certain stiffness during deformation. As the two substrates of the slit-pore model are separated by the pore space, they can move freely in the normal direction so that we add a linear spring of a force constant $k$ connecting the two solid substrates to account for the stiffness in the normal direction. Specifically, the spring connects the centers of mass (COM) of the two outer layers of solid atoms and only accounts for the relative displacement in the normal direction. One advantage in doing this is that one can reproduce any target modulus in the normal direction by tuning the spring constant $k$. In fact, $k$ can be related to Young's modulus in the normal direction $E_{z z}$ :

$$
E_{z z}=\frac{k L_{z}}{L_{x} L_{y}}
$$

where $L_{\mathrm{x}}, L_{\mathrm{y}}, L_{\mathrm{z}}$ are the sizes of the solid model in the $x, y, z$ directions, respectively. 


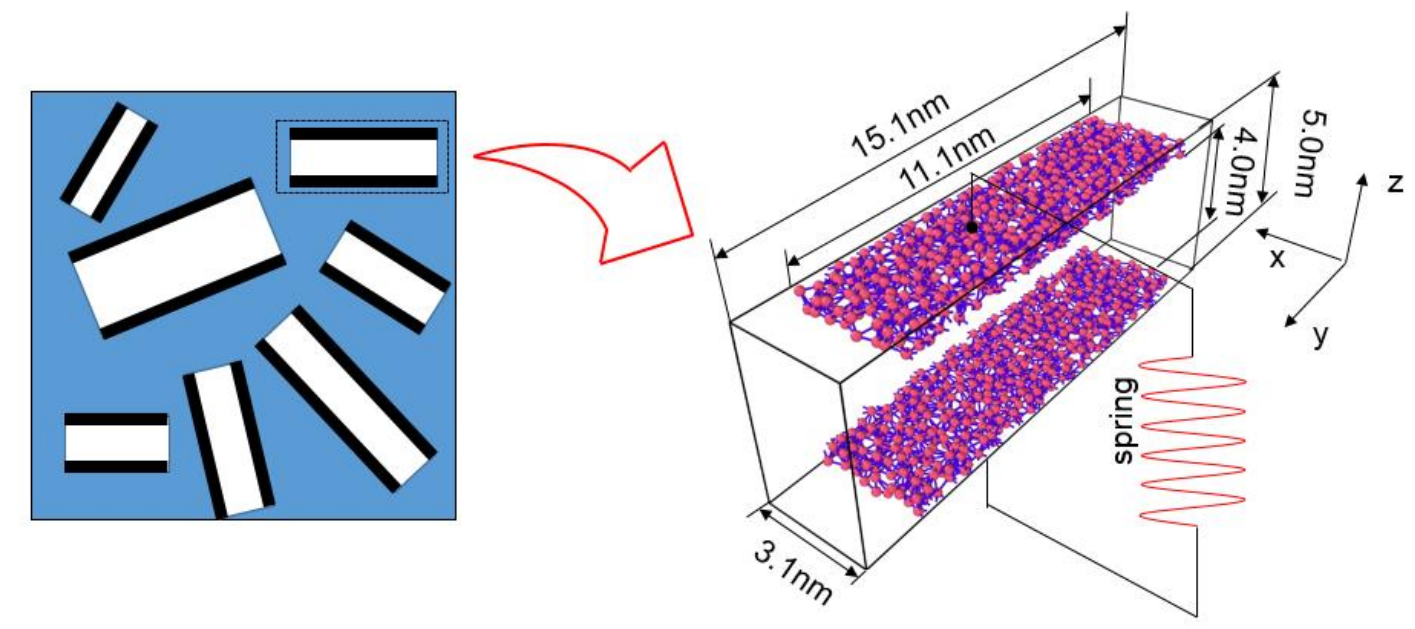

Figure 1. Deformable atomistic slit pore model used in the present work. The slit pore model (right) is assumed to be taken from a linear elastic porous medium containing slit pores (left). The mechanical effect of surrounding material is simply described using a spring connecting the two substrates. The longitudinal and normal directions are defined as the $y$ and $z$ directions, respectively.

\section{Simulation details use subsection numbering}

As both sorption and deformation arise at the molecular scale, we use hybrid molecular simulations including both Grand Canonical Monte Carlo (GCMC) and Molecular Dynamics (MD). The GCMC method, which is often used to determine sorption isotherms in various materials, fails to model the sorption-induced deformation directly as it relies on the Grand Canonical ensemble which imposes a constant volume (and therefore does not allow deformation). On the other hand, MD allows the deformation of the material but cannot model the sorption process. Thus, we employ a hybrid GCMC/MD method, which runs iterations of GCMC and MD simulations until an equilibrium state is reached. Specifically, each block of GCMC and MD includes 1000 trial insertion/deletion attempts of the fluid molecule and 1000 steps of Verlet integration of MD. In this work, $10^{5}$ blocks are carried out to reach equilibrium. 
Argon is taken as a simple prototypical adsorbate model and is described using the 12-6 Lennard-Jones $(\mathrm{LJ})$ potential with $\sigma_{\mathrm{ff}}=0.3405 \mathrm{~nm}$ and $\varepsilon_{\mathrm{ff}} / k_{\mathrm{B}}=119.8 \mathrm{~K}\left(k_{\mathrm{B}}\right.$ is the Boltzmann constant). The solid-fluid interaction is also modeled using the $\mathrm{LJ}$ potential with $\sigma_{\mathrm{sf}}=0.45 \mathrm{~nm}$ and $\varepsilon_{\mathrm{sf}}=2 \varepsilon_{\mathrm{ff}}$. The mechanical properties of the solid are solely controlled by the harmonic bonds and the spring described above. The sorption process is carried out at a constant temperature $T=87 \mathrm{~K}$. The simulation box boundaries are made periodic in all the three directions of space. In the $y$ and $z$ directions, some void is left between the substrates and the box boundary to allow the deformation. However, this additional space is not accessible for the argon atoms so that it cannot be considered that the stress imposed in this direction corresponds to the argon gas pressure imposed through the use of constant chemical potential simulations. I modified the previous sentence, please check that it is ok. To prevent any tangential drift of the upper and lower solid plates during the simulation, the center-of-mass position of each solid plate is constrained along the tangential direction ( $y$ and $z$ direction) by adjusting the coordinates of the atoms every timestep while maintaining relative coordinates of any pair of atoms in the solid plates.

\section{Results and discussion}

\section{Sorption and strain isotherms}

The sorption isotherms are shown in Figure 2 (a,top). Starting from an empty pore, upon increasing the pressure, adsorption starts with a film forming at the solid wall at low relative pressure (Figure $2 \mathrm{~b}(\mathrm{I})$ ) - this domain is referred to as "film region" in what follows. Capillary condensation (Figure $2 \mathrm{~b}$ (II)) occurs at a certain chemical potential at which the vapor phase between the liquid films is replaced by a liquid phase forming menisci - this domain is referred to as "filled region". Afterwards, the adsorption amount increases gradually as the menisci at the two open ends flatten. On the other hand, desorption starts with the retreating of menisci (Figure $2 b($ III)) until the fluid separates at a certain relative pressure and only adsorbed films are left on the solid walls. This process is referred to as 
capillary evaporation. The difference in the chemical potential at which capillary condensation and evaporation occur results in a hysteresis loop in the sorption isotherm. The simulated sorption behavior is consistent with experiments and theories conducted by other researchers ${ }^{3637}$.
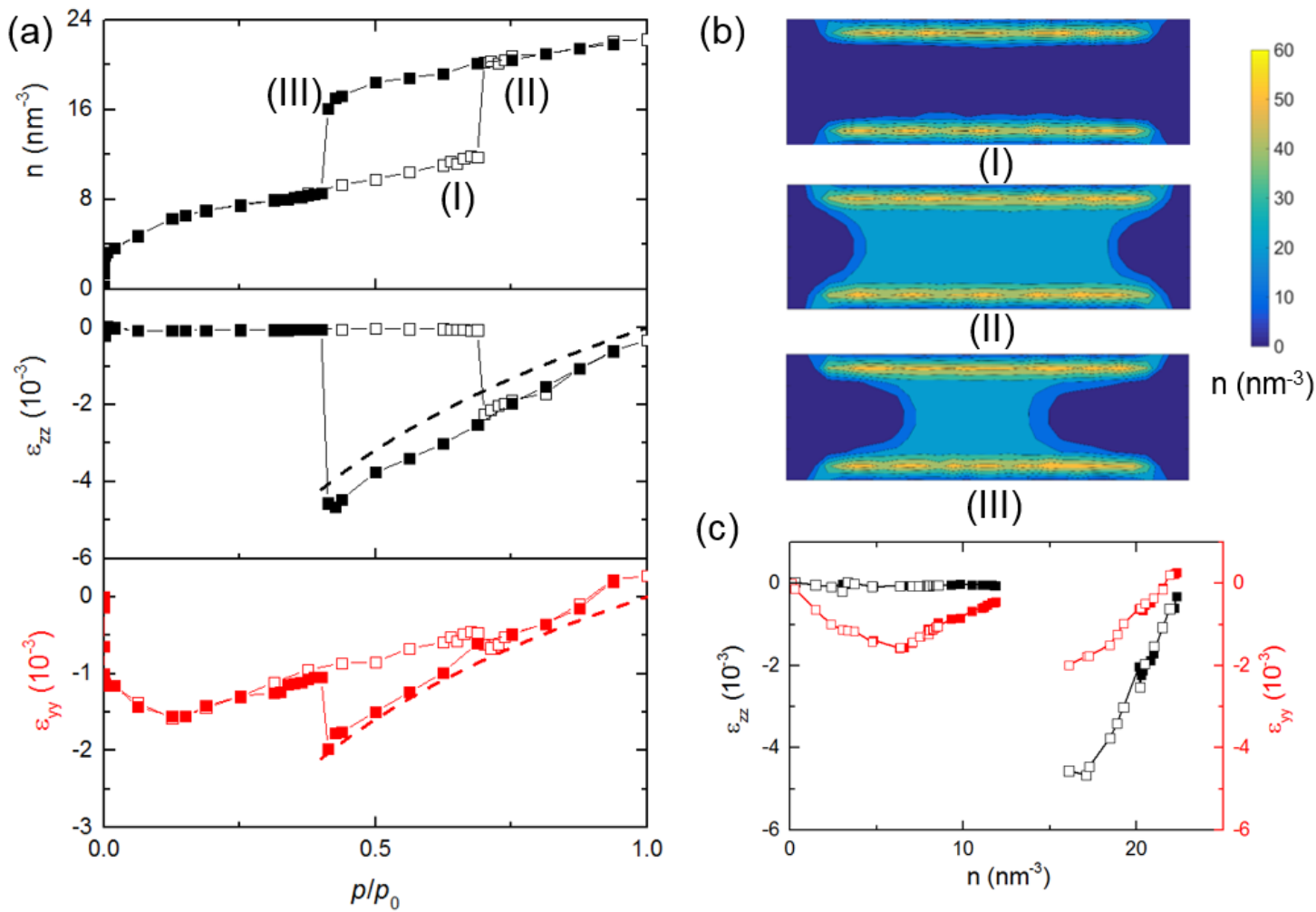

Figure 2. (a) Sorption isotherms (top), normal strain (center) and longitudinal strain (bottom) as observed from molecular simulation. The adsorbed amount is given in pore density $\mathrm{n}$ which is defined as $\mathrm{n}=\mathrm{N} / \mathrm{V}$, where $\mathrm{N}$ and $\mathrm{V}$ are the number of adsorbed molecules and pore volume, respectively. The open and closed symbols represent the adsorption and desorption branches, respectively. The dashed lines are the strain isotherms in the filled region predicted using Eq. (3). (b) Fluid density map corresponding to points I, II, III in (a); (c) Strains $\varepsilon_{z z}$ and $\varepsilon_{y y}$ as a function of adsorbed amount $\mathrm{n}$. The black and red symbols correspond to the normal strain $\varepsilon_{z z}$ and longitudinal strain $\varepsilon_{y y}$, respectively. The open and closed symbols represent the adsorption and desorption branches, respectively. 
Sorption results in deformations in the normal and longitudinal directions as shown in Figure 2 (a) (center and bottom). In the normal direction, no significant deformation is observed in the film region during adsorption until condensation occurs where an abrupt shrinkage occurs along the phase transition. In the filled region, as the pressure keeps increasing, the shrinkage reverts back to zero strain as the menisci flatten. Upon desorption, shrinkage increases in a reversible fashion as the relative pressure decreases until evaporation occurs. Afterwards, as the pressure keeps decreasing, the strain goes back to zero in the film region. In the longitudinal direction, shrinkage is observed even at very low relative pressures in the film region and reaches a maximum at a point around 0.15 $p / p_{0}$. Then, shrinkage decreases as the relative pressure keeps increasing in the film region (the origin of the maximum in the longitudinal shrinkage will be discussed below). A small yet abrupt shrinkage is observed at the condensation point. In the filled region, the longitudinal strain increases back to zero in a fashion similar to that observed for the normal strain. Upon desorption, the longitudinal strain decreases beyond the condensation point and jumps at the evaporation point back to the strain observed upon adsorption in the film region.

Altogether, the results above show that the system deforms quite differently in the normal and longitudinal directions in the film region while both normal and longitudinal strains follow the same trend in the filled region. A pronounced hysteresis is observed in both the normal strain and longitudinal strain isotherms. Obviously, this hysteresis is concomittant with the hysteresis observed in the sorption isotherm. However, no hysteresis is found when plotting the strain as a function of the adsorbed amount in Figure 2(c), therefore showing that the strain can be unambiguously defined for a given fluid configuration.

\section{Deformation mechanisms}

For the normal direction, the deformation is driven by the internal normal pressure of the fluid, $p_{\mathrm{zz}}$. In the film region, the two films are separated by a vapor phase which is in contact 
with the vapor reservoir located outside the porosity. Considering the force balance in the $\mathrm{z}$-direction, we get $p_{z z} \sim p$ where $p$ is the vapor pressure in the reservoir. Thus, the maximum vapor pressure that can be achieved in the film region is around $6000 \mathrm{~Pa}$, which corresponds to the condensation pressure. Given the modulus $E_{\mathrm{zz}}=5 \mathrm{GPa}$ used to maintain the two pore surfaces at a distance corresponding to the nominal pore size (see the definition of the spring used in the section method), the expected strain in the film region $\varepsilon=p / E_{z z}<1.2 \mathrm{E}-6$ please use scientific notation for Langmuir not engineering notation: $1 \mathrm{E}-6$ should be $10^{-6}$ is negligible in agreement with the values obtained in our molecular simulations. However, in the filled region, the pore space is filled with a closely packed liquid phase so that two mechanical effects arise: (1) the meniscus formed at the open ends imposes a Laplace pressure on the liquid and (2) the packing behavior of the atoms in the liquid phase can introduce a disjoining pressure term. The pressure due to the meniscus is given by the Laplace pressure:

$$
p_{V}-p_{L}=\frac{R_{g} T}{V_{L}} \ln \frac{p_{V}}{p_{0}}
$$

where $R_{g}$ is the ideal gas constant, $V_{L}$ is the molar volume of the condensed phase, $T$ is the temperature, $p_{V}$ and $p_{L}$ are the pressures of the vapor and liquid phases, respectively. Under the condition of linear elasticity and small deformation, the strain in the normal direction caused by the Laplace pressure can be calculated as:

$$
\varepsilon_{z z}^{L}=\frac{p_{V}-p_{L}}{E_{z z}}=\frac{R_{g} T}{V_{L} E_{z z}} \ln \frac{p_{V}}{p_{S A T}}
$$

As shown in Figure 2 (a), this estimated $\varepsilon_{z z}^{L}$ is in reasonable agreement with the simulated normal strain but with a constant offset. This indicates that the variation of strain with the relative pressure in the filled region is mainly driven by the variation of the Laplace pressure. The constant offset in Figure 2 (a), which corresponds to an additional term arising from the liquid pressure, is thought to be due to the disjoining pressure. Generally the disjoining pressure is strongly pore size dependent and decays while oscillating upon increasing the pore size. It indicates that in our simulation the disjoining pressure is negative so that further shrinkage with respect to the Laplace pressure effect applies. Note 
that this disjoining pressure only persists in the filled region as the surfaces see each other through the liquid phase. In contrast, in the film region, the two films are separated by the vapor phase so that they do not interact with each other and the disjoining pressure becomes negligible (we note that the pore size is larger than the interaction cutoff used in our simulations so that no disjoining effect is expected in the gas phase).

For the longitudinal direction, significant deformation is observed in the film region. This is generally explained by the surface stress. However, a detailed analysis of the strain isotherm at very low relative pressure indicates that shrinkage starts at the very beginning of the adsorption process - i.e. even before a single-layer film is formed. As shown in Figure $3 \mathrm{a}$, the strain decreases (increasing shrinkage) with the adsorbed amount with a steep initial slope, therefore indicating that the effect of scattered fluid atoms on the deformation of the substrates can be very significant. Figure $3 \mathrm{~b}$ illustrates the film formation process of the first layer, showing the densification of the adsorbed atoms at the solid surface. More specifically, the adsorbed fluid atoms make the solid shrink to maximize the density of fluid-solid interactions.

(a)

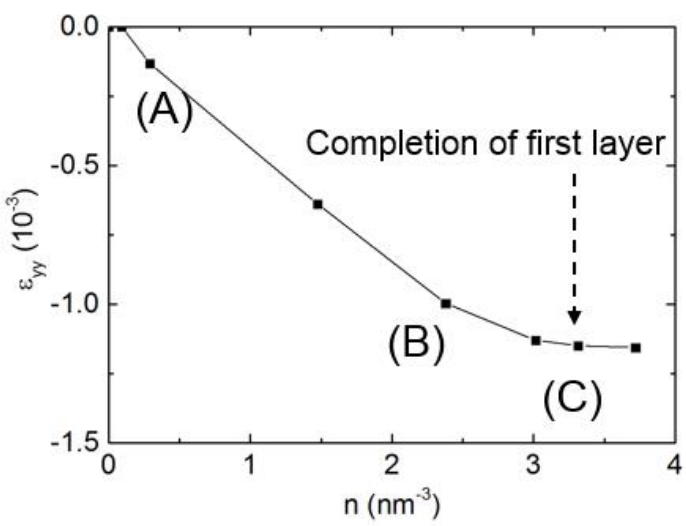

(b)

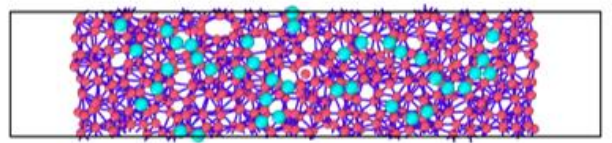

(A)

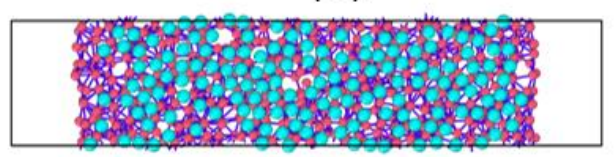

(B)

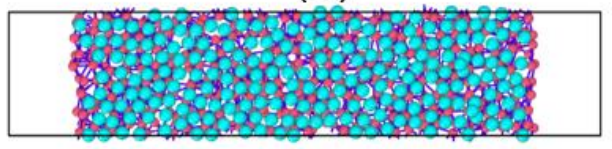

(C)

Figure 3. (a) Longitudinal strain as a function of pore fluid density before the completion of first adsorbed layer. (b) Typical molecular configuration showing the adsorbed film at points (I) (II) (III) in (a). 
To further illustrate the longitudinal shrinkage observed in the film region, we consider the simple following model where adsorbed atoms interact with solid substrate as shown in Figure 4(a). We use in this model the same solid-liquid Lennard-Jones interaction potential used in the molecular simulations. The initial distance between nearest neighbor solid atoms is taken to be $0.38 \mathrm{~nm}$. Because of the additivity of the energy for liquid-solid pair interaction potentials, the energy of the system can be expressed as the sum of solid-solid, solid-liquid and liquid-liquid energy terms:

$$
\begin{aligned}
U & =U_{\mathrm{SS}}\left(\varepsilon_{y y}\right)+U_{\mathrm{SL}}\left(\varepsilon_{y y}\right)+U_{\mathrm{LL}}\left(\varepsilon_{y y}\right) \\
& =C \varepsilon_{y y}^{2}+4 \varepsilon_{\mathrm{sf}} \sum_{i}\left(\frac{\sigma_{\mathrm{sf}}^{12}}{R_{i}^{12}}-\frac{\sigma_{\mathrm{sf}}^{6}}{R_{i}^{6}}\right)+4 \varepsilon_{\mathrm{ff}}\left[\frac{\sigma_{\mathrm{ff}}^{12}}{(\omega a)^{12}}-\frac{\sigma_{\mathrm{ff}}^{6}}{(\omega a)^{12}}\right]
\end{aligned}
$$

Here the solid-solid, solid-liquid and liquid-liquid energy are $U_{\mathrm{SS}}, U_{\mathrm{SL}}$ and $U_{\mathrm{LL}}$. The solid is assumed to behave in a linear elastic fashion so that $U_{\text {ss }}$ is expressed as a quadratic strain term, $C \varepsilon_{y y}^{2}$, where $C$ is the elastic constant. Both $U_{\mathrm{SL}}$ and $U_{\mathrm{LL}}$ are expressed in terms of a $\mathrm{LJ}$ potential. The distance between the $i$-th solid atom and the liquid atom is $R_{\mathrm{i}}$ while the distance between the neighboring solid atoms is $a$. For the sake of simplicity, the distance between the neighboring gas atoms is written $\omega a$ where $\omega$ is the distance ratio between gas atom distance and solid atom distance. By minimizing $U$, one can get $\varepsilon_{y y}$ at equilibrium. Let us now consider the very simple situation where only one liquid atom is adsorbed at the solid surface. As shown in Figure 4 (b), the solid-liquid interaction energy (black curve) increases with the strain, which indicates that the system can reach lower energy level upon shrinking. Now if we take into account the contribution of the elastic energy (blue curve), we find an energy minimum at a negative longitudinal strain, which indicates that the solid undergoes shrinkage because of the solid-fluid interaction. Another important factor is the 2D internal film pressure which arises from the fluid-fluid interaction energy $U_{\mathrm{ff}}$. At very low relative pressure, the argon atoms are far from each other which results in small attractive inter-molecular forces and therefore a relatively small negative pressure contribution. Considering the force balance in the longitudinal direction, the negative fluid pressure will cause additional shrinkage of the solid substrate as indicated by the solid red 
line $(\omega=5)$ in Figure 4(b). As the relative pressure increases, the adsorbed argon film becomes denser ( $\omega$ decreases) and the amplitude of the attractive inter-molecular forces becomes larger; as a result, the liquid pressure become more negative which leads to a larger shrinkage strain in the solid substrate (dashed red line, $\omega=3$ in Figure 4 (b)). However, if the film density keeps increasing, the attractive inter-molecular force decreases and eventually becomes repulsive, resulting into a swelling strain of the solid substrate $(\omega=1$, dotted red line in Figure 4(b)).

In the filled region, the longitudinal strain is found to follow a similar trend with that of the normal direction: abrupt shrinkage at condensation and evaporation points. The magnitude of shrinkage decreases as the relative pressure increases, which indicates that the Laplace pressure also takes effect in the longitudinal direction (Eq. (5) also applies by substituting $E_{\mathrm{zz}}$ with $\left.E_{\mathrm{yy}}\right)$.

(a)

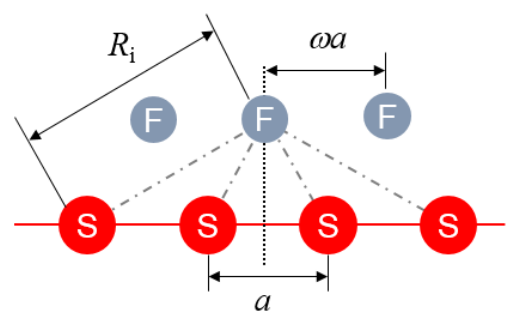

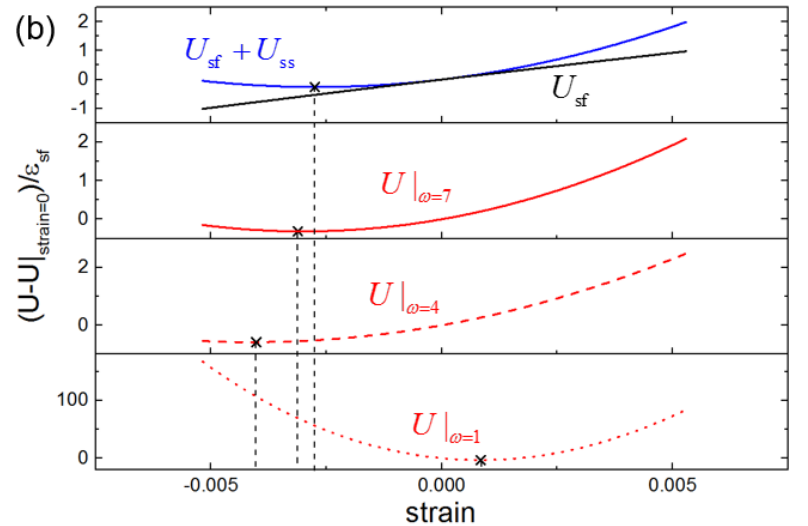

Figure 4. (a) Schematic view of a system consisting of a solid substrate and adsorbed gas atoms. G and S represent gas and solid atoms respectively. The solid-solid, solid-gas, gasgas distances are $a, R_{\mathrm{i}}$, and $\omega a$. (b) Relationship between the system energies and the tangential strain at different film density. The tangential strain at equilibrium corresponds to the minimal energy at each density. As the film density increases, the strain can turn 
from negative to positive in agreement with the molecular simulation reported in the present work. I have a problem with your drawing because $\mathrm{wa}=\mathrm{a}$ so that $\mathrm{w}=1$. You need to plot a figure that better illustrates what you have in mind. Otherwise it looks like you 11 always have $\mathrm{w}=1$. Can you plot something that shows $\mathrm{w}$ different from 1 ?

\section{Isosteric enthalpy of adsorption Again use subsection numbering}

The sorption-induced deformation of the slit pore can also be investigated by checking the isosteric enthalpy of adsorption of the system along the adsorption/strain isotherm:

$$
q_{s t}=\langle E\rangle(\langle N\rangle)-\langle E\rangle(\langle N\rangle+1)=q_{s t}^{S L}+q_{s t}^{L L}+q_{s t}^{S S}
$$

where $E$ is the potential energy of the system and $N$ is the adsorbed amount. Because of the additivity of the energy, the isosteric heat of adsorption can be split into three contributions: The solid-fluid $q_{s t}^{S L}$, fluid-fluid $q_{s t}^{L L}$ and solid-solid $q_{s t}^{S S}$ contributions. There $\mathrm{s}$ something really wrong here. You can simplify by qst $=-\langle E\rangle$ which is obviously not correct. Is this what you did? You need to use the following formula:

$$
q_{\mathrm{st}}=R T-\frac{\partial\langle U\rangle}{\partial\langle N\rangle}=R T-\frac{\langle U N\rangle-\langle U\rangle\langle N\rangle}{\left\langle N^{2}\right\rangle-(\langle N\rangle)^{2}}
$$

According to Fig. $5 q_{s t}^{S L}$ decreases and $q_{s t}^{L L}$ increases with increasing the adsorbed amount until a plateau is reached. These results are consistent with the data reported by other researchers. This can be attributed to the fact that at low adsorbed amount the enthalpy change associated with adsorption mainly arises from the contribution of solid-fluid interaction as the fluid molecules are adsorbed at the solid substrate. On the other hand, at larger adsorbed amounts, the enthalpy change mainly arises from the fluid-fluid interaction. The deformation of the system can be checked by looking at $q_{s t}^{S S}$ since it characterizes the change of deformation energy with adsorbed amount. According to Figure 5, $q_{s t}^{S S}$ is relatively small compared to the other two contributions, which indicates that the effect of deformation on the sorption process is not significant. On the other hand, we find a larger 
contribution of $q_{s t}^{S S}$ in the filled region as shown in the inset of Figure 5. This can be attributed to the larger dependence of the strain on the adsorbed amount (larger $\partial \varepsilon / \partial \mathrm{N})$. Considering the linear elasticity of the solid substrates, $q_{s t}^{S S}$ should satisfy

$$
q_{s t}^{S S} \approx-\frac{\partial U^{S S}}{\partial N}=-\frac{1}{2} V \sum_{x, y, z} E_{i i} \frac{\partial \varepsilon_{i i}^{2}}{\partial N}
$$

As shown in Figure 2 (c), the slope is steeper in the filled region which results in a larger $q_{s t}^{S S}$.

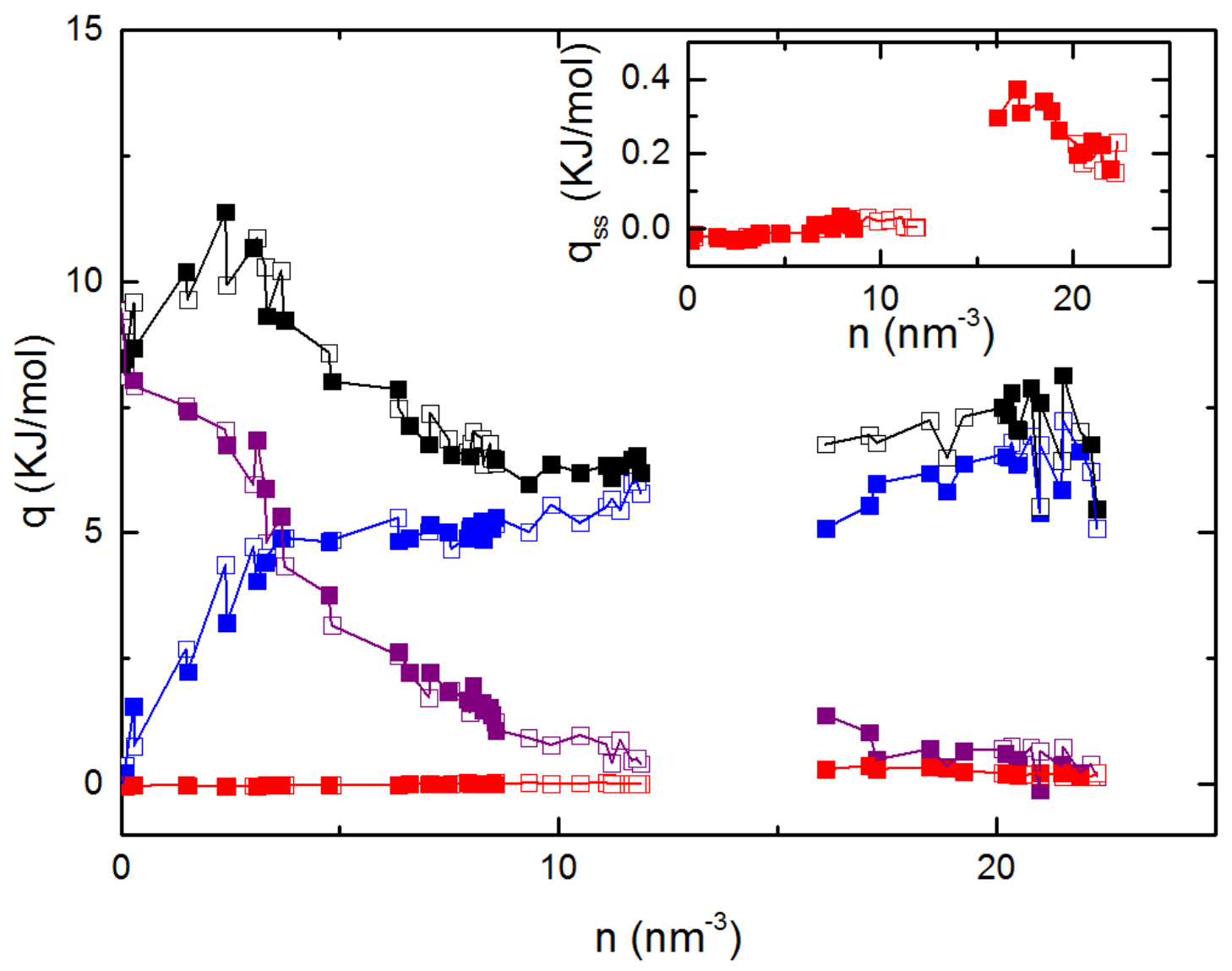

Figure 5. Different contributions to the isosteric enthalpy of adsorption as a function of the adsorbed amount $\mathrm{n}$. The black, purple, blue, red symbols represent $q_{s t}, q_{s t}^{S L}, q_{s t}^{L L}, q_{s t}^{S S}$ 
respectively. The inset shows a zoom-in the data for $q_{s t}^{S S}$. The open and closed symbols represent the adsorption and desorption data, respectively.

\section{Effect of temperature and solid/fluid interaction strength}

We further discuss the influence of two factors on the sorption-induced deformation: the solid-fluid interaction strength and the temperature. The solid-fluid interaction strength is tuned by changing the solid-fluid bonding energy $\varepsilon_{\mathrm{sf}}$ in the LJ potential. As shown in Figure 6(a), the solid-fluid interaction does not strongly affect the normal strain. This can be attributed to the fact the normal strain is mainly governed by the Laplace pressure, which is independent of solid-liquid interaction as a first order approximation. On the contrary, the solid-liquid interaction significantly influences the longitudinal strain in the film region. More in detail, a stronger solid-liquid strength leads to larger shrinkage magnitude. As the shrinkage is caused by the attractive force between the solid and liquid atoms, a stronger solid-liquid strength induces larger attractive forces, resulting in larger shrinkages in the film region. On the other hand, as the longitudinal strain is governed by Laplace pressure in the filled region, the influence of the solid-fluid interaction becomes negligible in the bulk phase region. The temperature plays an important role on the normal strain by influencing the Laplace pressure (Figure 6b). We find that the simulated strain is consistent with the values predicted from Eq. (3). For the longitudinal strain, the temperature does not have a significant influence in the film region. Although the change of temperature influences the liquid pressure, the strain is mainly governed by the solid-fluid interaction and the virial part of the liquid pressure. On the other hand, the influence of the temperature on the longitudinal strain in the bulk phase region is similar to that on the normal strain as they are both governed by the Laplace pressure. 
(a)

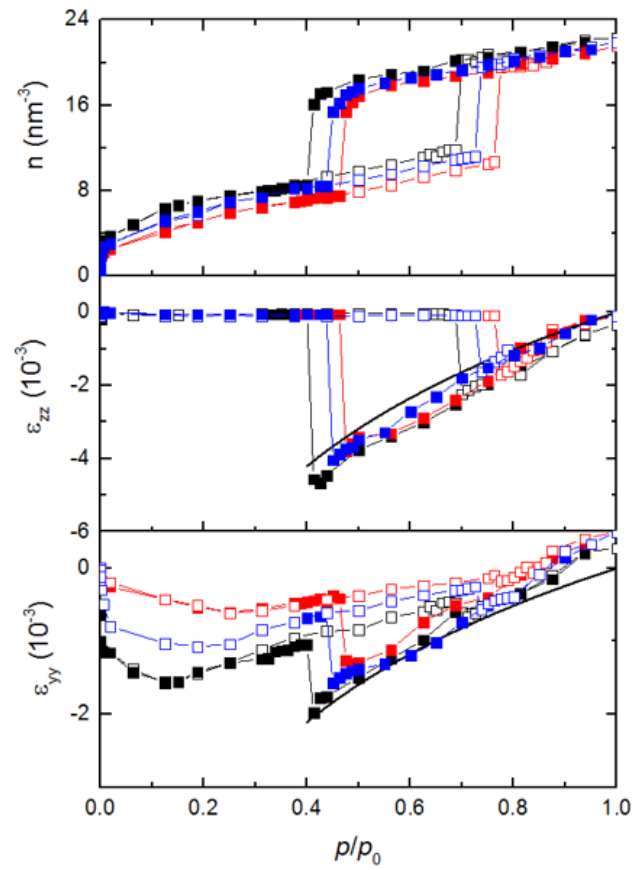

(b)

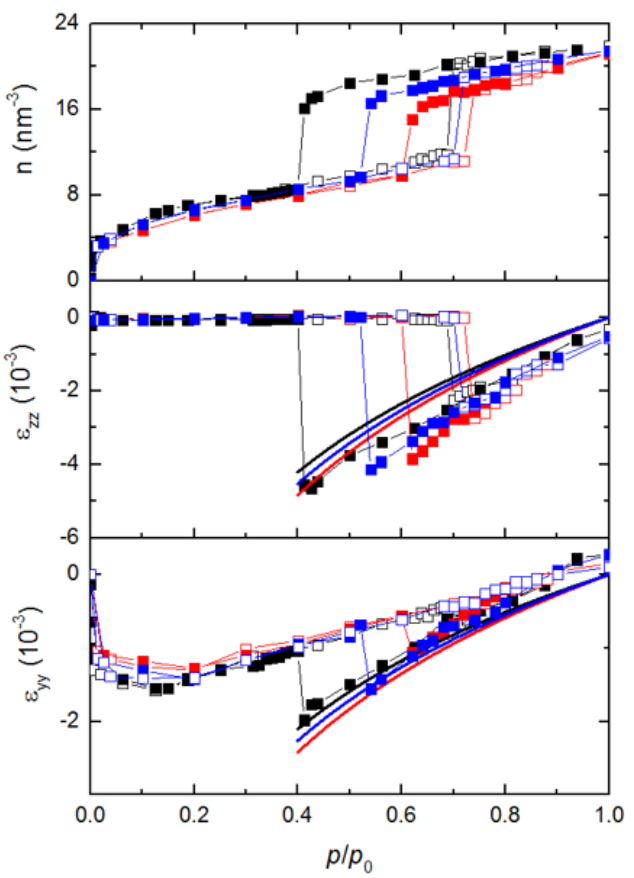

Figure 6. Effect of different factors on the strain isotherm. (a) Effect of solid-fluid interaction strength: the red, blue and black symbols represent different interaction strengths: $\varepsilon_{\mathrm{sf}}=\varepsilon_{\mathrm{ff}}, 1.5 \varepsilon_{\mathrm{sf}}$ and $2.0 \varepsilon_{\mathrm{sf}}$, respectively. The open and closed symbols represent the adsorption and desorption data, respectively. The black solid line is the strain isotherm as predicted by Eq. (5). (b) Effect of temperature: the red, blue and black symbols represent data for $T=100 \mathrm{~K} 92.5 \mathrm{~K}$ and $87 \mathrm{~K}$. The open and closed symbols represent the adsorption and desorption data, respectively. The red and black solid lines are the strain isotherms as predicted by Eq.(5) for $T=100 \mathrm{~K}$ and $87 \mathrm{~K}$, respectively.

\section{Conclusion}

In this work, the sorption-induced deformation in an atomistic slit pore is simulated. We find that the sorption-induced deformation is governed by different mechanisms depending on the adsorption region considered. In the liquid-filled region, the Laplace pressure is dominating both the longitudinal and normal strains. In contrast, in the film region, the normal strain can be neglected while the longitudinal strain is driven by both the solidliquid attractive forces and the 2D pressure in the liquid film. 


\section{Acknowledgements}

The authors acknowledge the support of the Swiss National Science Foundation (SNSF) (No.143601).

\section{References}

(1) Gor, G. Y.; Huber, P.; Bernstein, N. Adsorption-Induced Deformation of Nanoporous Materials-A Review. Appl. Phys. Rev. 2017, 4 (1), 011303. https://doi.org/10.1063/1.4975001.

(2) Kulasinski, K.; Guyer, R.; Keten, S.; Derome, D.; Carmeliet, J. Impact of Moisture Adsorption on Structure and Physical Properties of Amorphous Biopolymers. Macromolecules 2015, 48 (8), 2793-2800. https://doi.org/10.1021/acs.macromol.5b00248.

(3) Brochard, L.; Vandamme, M.; Pellenq, R. J. M.; Fen-Chong, T. AdsorptionInduced Deformation of Microporous Materials: Coal Swelling Induced by CO 2CH 4 Competitive Adsorption. Langmuir 2012, 28 (5), 2659-2670. https://doi.org/10.1021/la204072d.

(4) Neimark, A. V; Ravikovitch, P. I. Capillary Condensation in MMS and Pore Structure Characterization. Microporous Mesoporous Mater. 2001, 44-45, 697707. https://doi.org/10.1016/S1387-1811(01)00251-7.

(5) Shao, L.-H.; Jin, H.-J.; Viswanath, R. N.; Weissmüller, J. Different Measures for the Capillarity-Driven Deformation of a Nanoporous Metal. EPL (Europhysics Lett. 2010, 89 (6), 66001. https://doi.org/10.1209/0295-5075/89/66001.

(6) Prass, J.; Müter, D.; Fratzl, P.; Paris, O.; Mueter, D. Capillarity-Driven 
Deformation of Ordered Nanoporous Silica. Appl. Phys. Lett. 2009, 95 (8), 083121/1-083121/3. https://doi.org/10.1063/1.3213564.

(7) Balzer, C.; Morak, R.; Erko, M.; Triantafillidis, C.; H??sing, N.; Reichenauer, G.; Paris, O. Relationship between Pore Structure and Sorption-Induced Deformation in Hierarchical Silica-Based Monoliths. Zeitschrift fur Phys. Chemie 2015, 229 (78), 1189-1209. https://doi.org/10.1515/zpch-2014-0542.

(8) Gor, G. Y.; Paris, O.; Prass, J.; Russo, P. a.; Ribeiro Carrott, M. M. L.; Neimark, A. V. Adsorption of N-Pentane on Mesoporous Silica and Adsorbent Deformation. Langmuir 2013, 29 (27), 8601-8608. https://doi.org/10.1021/la401513n.

(9) Gor, G. Y.; Bernstein, N. Adsorption-Induced Surface Stresses of the Water/Quartz Interface: Ab Initio Molecular Dynamics Study. Langmuir 2016, 32 (21), 5259-5266. https://doi.org/10.1021/acs.langmuir.6b00923.

(10) Gor, G. Y.; Bernstein, N. Revisiting Bangham's Law of Adsorption-Induced Deformation: Changes of Surface Energy and Surface Stress. Phys. Chem. Chem. Phys. 2016, 18 (14), 9788-9798. https://doi.org/10.1039/C6CP00051G.

(11) Gor, G. Y.; Neimark, A. V. Adsorption-Induced Deformation of Mesoporous Solids: Macroscopic Approach and Density Functional Theory. Langmuir 2011, 27 (11), 6926-6931. https://doi.org/10.1021/la201271p.

(12) Kolesnikov, A.; Georgi, N.; Budkov, Y. A.; Möllmer, J.; Hofmann, J.; Adolphs, J. Effects of Enhanced Flexibility and Pore Size Distribution on Adsorption-Induced Deformation of Mesoporous Materials. Langmuir 2018. https://doi.org/10.1021/acs.langmuir.8b00591.

(13) Cornette, V.; de Oliveira, J. C. A.; Yelpo, V.; Azevedo, D.; López, R. H. Binary Gas Mixture Adsorption-Induced Deformation of Microporous Carbons by Monte 
Carlo Simulation. J. Colloid Interface Sci. 2018, 522, 291-298.

https://doi.org/10.1016/j.jcis.2018.03.026.

(14) Grégoire, D.; Malheiro, C.; Miqueu, C. Estimation of Adsorption-Induced Pore Pressure and Confinement in a Nanoscopic Slit Pore by a Density Functional Theory. Contin. Mech. Thermodyn. 2017, 30 (2), 1-17. https://doi.org/10.1007/s00161-017-0602-x.

(15) BANGHAM, D. H.; FAKHOURY, N. The Expansion of Charcoal Accompanying Sorption of Gases and Vapours. Nature 1928, 122 (3079), 681-682. https://doi.org/10.1038/122681b0.

(16) Shuttleworth, R. The Surface Tension of Solids. Proc. Phys. Soc. Sect. A 1950, 63 (5), 444-457. https://doi.org/10.1088/0370-1298/63/5/302.

(17) Zhang, Y. Mechanics of Adsorption-deformation Coupling in Porous Media. $J$. Mech. Phys. Solids 2018, 114, 31-54. https://doi.org/10.1016/j.jmps.2018.02.009.

(18) Steele, W. A.; Halsey, G. D. The Interaction of Gas Molecules with Capillary and Crystal Lattice Surfaces. J. Phys. Chem. 1955, 59 (1), 57-65. https://doi.org/10.1021/j150523a017.

(19) Stoeckli, F. The Gas-Solid Interface Calculations of Adsorption Potentials in SlotLike Pores of Molecular Dimensions. Helv. Chim. Acta 1974, 57 (7), 2195-2199. https://doi.org/10.1002/hlca.19740570734.

(20) Derjaguin, B. V.; Churaev, N. V. Polymolecular Adsorption and Capillary Condensation in Narrow Slit Pores. Prog. Surf. Sci. 1992, 40 (1-4), 173-191. https://doi.org/10.1016/0079-6816(92)90045-J.

(21) Coasne, B. Multiscale Adsorption and Transport in Hierarchical Porous Materials. 
New J. Chem. 2016, 40 (5), 4078-4094. https://doi.org/10.1039/C5NJ03194J.

(22) Balbuena, P. B.; Gubbins, K. E. Classification of Adsorption Behavior: Simple Fluids in Pores of Slit-Shaped Geometry. Fluid Phase Equilib. 1992, 76 (C), 2135. https://doi.org/10.1016/0378-3812(92)85075-J.

(23) Suzuki, T.; Kaneko, K.; Setoyama, N.; Maddox, M.; Gubbins, K. Grand Canonical Monte Carlo Simulation for Nitrogen Adsorption in Graphitic Slit Micropores: Effect of Interlayer Distance. Carbon N. Y. 1996, 34 (7), 909-912. https://doi.org/10.1016/0008-6223(96)00049-8.

(24) Jagiello, J.; Olivier, J. P. 2D-NLDFT Adsorption Models for Carbon Slit-Shaped Pores with Surface Energetical Heterogeneity and Geometrical Corrugation. Carbon N. Y. 2013, 55 (2), 70-80. https://doi.org/10.1016/j.carbon.2012.12.011.

(25) Striolo, A.; Chialvo, A. A.; Cummings, P. T.; Gubbins, K. E. Water Adsorption in Carbon-Slit Nanopores. Langmuir 2003, 19 (20), 8583-8591. https://doi.org/10.1021/la0347354.

(26) Long, Y.; Palmer, J. C.; Coasne, B.; Śliwinska-Bartkowiak, M.; Gubbins, K. E. Pressure Enhancement in Carbon Nanopores: A Major Confinement Effect. Phys. Chem. Chem. Phys. 2011, 13 (38), 17163. https://doi.org/10.1039/c1cp21407a.

(27) Long, Y.; Palmer, J. C.; Coasne, B.; Śliwinska-Bartkowiak, M.; Gubbins, K. E. Under Pressure: Quasi-High Pressure Effects in Nanopores. Microporous Mesoporous Mater. 2012, 154, 19-23. https://doi.org/10.1016/j.micromeso.2011.07.017.

(28) Long, Y.; Palmer, J. C.; Coasne, B.; Śliwinska-Bartkowiak, M.; Jackson, G.; Müller, E. A.; Gubbins, K. E.; Śliwinska-Bartkowiak, M.; Jackson, G.; Müller, E. a.; et al. On the Molecular Origin of High-Pressure Effects in Nanoconfinement: 
The Role of Surface Chemistry and Roughness. J. Chem. Phys. 2013, 139 (14), 144701. https://doi.org/10.1063/1.4824125.

(29) Diao, R.; Fan, C.; Do, D. D.; Nicholson, D. Monte Carlo Simulation of Adsorption-Induced Deformation in Finite Graphitic Slit Pores. J. Phys. Chem. C 2016, 120 (51), 29272-29282. https://doi.org/10.1021/acs.jpcc.6b10135.

(30) Diao, R.; Fan, C.; Do, D. D.; Nicholson, D. Adsorption Induced Deformation in Graphitic Slit Mesopores: A Monte Carlo Simulation Study. Chem. Eng. J. 2017, 328, 280-292. https://doi.org/10.1016/j.cej.2017.07.013.

(31) Balzer, C.; Cimino, R. T.; Gor, G. Y.; Neimark, A. V.; Reichenauer, G. Deformation of Microporous Carbons during N2, Ar, and CO2Adsorption: Insight from the Density Functional Theory. Langmuir 2016, 32 (32), 8265-8274. https://doi.org/10.1021/acs.langmuir.6b02036.

(32) Günther, G.; Schoen, M. Sorption Strain as a Packing Phenomenon. Phys. Chem. Chem. Phys. 2009, 11 (40), 9082. https://doi.org/10.1039/b903514a.

(33) Günther, G.; Schoen, M. Sorption Strains and Their Consequences for Capillary Condensation in Nanoconfinement. Mol. Simul. 2009, 35 (1-2), 138-150. https://doi.org/10.1080/08927020802412370.

(34) Schoen, M.; Günther, G. Phase Transitions in Nanoconfined Fluids: Synergistic Coupling between Soft and Hard Matter. Soft Matter 2010, 6 (23), 5832. https://doi.org/10.1039/c0sm00440e.

(35) Schoen, M.; Günther, G. Capillary Condensation in Deformable Mesopores: Wetting versus Nanomechanics. Mol. Phys. 2011, 109 (1), 83-95. https://doi.org/10.1080/00268976.2010.513346. 
(36) Horikawa, T.; Do, D. D.; Nicholson, D. Capillary Condensation of Adsorbates in Porous Materials. Adv. Colloid Interface Sci. 2011, 169 (1), 40-58. https://doi.org/10.1016/j.cis.2011.08.003.

(37) Thommes, M.; Cychosz, K. A. Physical Adsorption Characterization of Nanoporous Materials: Progress and Challenges. Adsorption 2014, 20 (2-3), 233250. https://doi.org/10.1007/s10450-014-9606-z. 